Itinéraires Itinéraires

Littérature, textes, cultures

2016-2 | 2017

Livre, sérialité et transmédialité

\title{
Évolution de la place du livre dans le circuit transmédiatique du media mix japonais : le cas
} Dragon Ball

Evolution of the Role of the Book in the Japanese Media Mix. The Example of Dragon Ball

\section{Bounthavy Suvilay}

\section{OpenEdition}

Journals

Édition électronique

URL : http://journals.openedition.org/itineraires/3429

DOI : $10.4000 /$ itineraires.3429

ISSN : 2427-920X

Éditeur

Pléiade

\section{Référence électronique}

Bounthavy Suvilay, «Évolution de la place du livre dans le circuit transmédiatique du media mix japonais : le cas Dragon Ball », Itinéraires [En ligne], 2016-2 | 2017, mis en ligne le 11 mai 2017, consulté le 02 octobre 2020. URL : http://journals.openedition.org/itineraires/3429 ; DOI : https://doi.org/ 10.4000/itineraires.3429

Ce document a été généré automatiquement le 2 octobre 2020.

\section{(c) (†) $\ominus$}

Itinéraires est mis à disposition selon les termes de la licence Creative Commons Attribution - Pas d'Utilisation Commerciale - Pas de Modification 4.0 International. 


\title{
Évolution de la place du livre dans le circuit transmédiatique du media mix japonais : le cas Dragon Ball
}

\author{
Evolution of the Role of the Book in the Japanese Media Mix. The Example of \\ Dragon Ball
}

Bounthavy Suvilay

1 Anne Besson (2015) utilise le terme de "constellations» en titre de son ouvrage consacré aux mondes fictifs contemporains, tandis que Richard Saint-Gelais emploie l'expression «modèle satellitaire » $(2011: 313)$ pour désigner les ensembles de fictions transfuges. Dans les discours du marketing, ces images astronomiques correspondent au terme « licence » qui désigne les fictions produites par les industries culturelles. Si le fonctionnement des franchises ${ }^{1}$ occidentales est relativement connu (Johnson 2013), il y a encore peu de recherches sur les licences japonaises ${ }^{2}$. Marc Steinberg (2012) analyse l'exemple historique de Tetsuwan Atom ${ }^{3}$ pour expliquer la formation d'une synergie multisectorielle afin de produire une série d'animation télévisée. Il nous semble utile d'analyser de manière littéraire un cas précis de media mix ${ }^{4}$ afin de montrer qu'il ne s'agit ni d'une simple traduction intersémiotique du livre vers d'autres supports ni d'une construction d'un monde diégétique cohérent à travers l'assemblage complémentaire de fictions transmédiatiques ${ }^{5}$. Dans ce dispositif, le livre est à la fois la source et la cible d'adaptations, le support de nouvelles extensions narratives ou la simple reprise d'une intrigue développée sur un autre média. En étudiant dans une perspective historique les liens entre ouvrages, séries animées, films et jeux vidéo de la licence Dragon Ball (DB), nous tenterons de montrer que la place du livre dans le circuit transmédiatique modifie les modalités de l'adaptation et témoigne de l'équilibre des médias dans un écosystème donné ${ }^{6}$. Le choix de ce corpus est lié à son succès en termes d'adaptation ${ }^{7}$ et à sa longévité qui permet de dresser un panorama de l'évolution des médias japonais et de mieux comprendre le fonctionnement de licences à succès actuelles comme One Piece ou Naruto ${ }^{8}$. Après une première phase de création parallèle du manga, de la série animée et des films, nous montrerons de quelle manière la fiction 
poursuit sa croissance dans le secteur du livre même si celui-ci n'est plus à l'initiative des séquences narratives. L'univers diégétique s'accroît par le biais de reformulations des mêmes intrigues pour des publics différents durant une période d'affaiblissement du secteur de l'édition papier. Mais le livre ne se contente pas d'être le relais des opérations de promotion et il apparaît de façon thématique dans le jeu vidéo où s'élabore désormais une partie de la fiction narrative.

\section{Du livre comme source}

\section{Un écosystème basé sur le papier}

2 Dans le circuit d'adaptation inauguré par Osamu Tezuka en 1963 comme dans la majorité des cas aujourd'hui encore, le support papier est à l'origine du dispositif media mix. Le processus concerne un récit qui est décliné sur d'autres supports : audiovisuels, vidéoludiques et ludiques. Ce dispositif donne une visibilité maximale au récit qui est matérialisé sur une multitude de supports à travers tous les réseaux de diffusion disponibles, la couverture médiatique assurant une captation de l'attention du public cible. Le système est utilisé pour les grands succès de librairie issus du magazine de l'éditeur Shueisha Shônen Jump, hebdomadaire qui avait une diffusion de 6,53 millions d'exemplaires à son pic d'audience en $1995^{\circ}$. Dans le cas de DB, ce dispositif media mix implique à la fois Shueisha (presse et livre), Toei Animation (série et films) et NamcoBandai (jeux vidéo, jouets) ${ }^{10}$.

3 La chronologie des médias étant optimisée pour que chaque secteur puisse bénéficier d'une rentabilisation idéale, il y a toujours un décalage entre le récit source (manga) et le récit cible (anime ${ }^{11}$ ) diffusé environ deux mois plus tard. Les événements du chapitre 327 de $D B$ (paru le 17 juin 1991) sont ainsi adaptés dans l'épisode 105 de DBZ (diffusé le 28 août 1991) ${ }^{12}$. Toei produit des épisodes hebdomadaires servant en grande partie à promouvoir le magazine de prépublication dont le récit est structurellement toujours en avance sur celui de l'anime. Les téléspectateurs avides de connaitre la suite $\mathrm{du}$ feuilleton deviennent presque mécaniquement des lecteurs, renforçant l'audience de l'éditeur papier, principal ayant droit de tous les produits liés à $D B$. Le media mix n'est donc pas un simple circuit d'adaptation multisectoriel mais surtout un réglage de lecture et de réception pour différents publics. La série étant considérée comme un produit d'appel, diffusée à la télévision afin de toucher un nouveau public et le convertir en lectorat payant du manga original, son scénario s'éloigne peu de celui du manga. Le format de vingt-cinq minutes est néanmoins trop long pour une transposition fidèle du manga. Comme l'explique Akira Toriyama (1999: 157), un chapitre transformé en anime dure à peine dix minutes. Il faut donc combler l'épisode avec un quart d'heure de séquences inédites nommées fillers afin de conserver la chronologie des médias et retarder l'avancée de l'anime $e^{13}$. Pour éviter ces expansions, la Toei et le diffuseur auraient pu concevoir une série comportant des épisodes plus courts. Néanmoins les pratiques toujours en cours favorisent les créneaux de 25 minutes pour fidéliser le public en créant un rendez-vous régulier ${ }^{14}$. Le contexte économique influe donc sur la production du récit, la présence de fillers étant liée au système de diffusion et au processus d'adaptation. 


\section{Interpolations et versions contrefictionnelles}

4 Les fillers introduisent deux types d'expansion, la première étant liée à la traduction intersémiotique d'un support à un autre, la seconde étant d'ordre transfictionnel. Le passage du manga à l'anime correspond à celui d'une fiction sous la forme du telling au showing (Hutcheon [2006] 2013) ou, si l'on se réfère à la classification de Marion (1997), d'un «média hétérochrone » (où le temps de réception n'est pas programmé par le support) à un support "homochrone", (incorporant le temps de réception dans l'énonciation de ses messages). En raison de cette gestion temporelle divergente, les séquences narratives des romans adaptés dans des médias homochrones sont plus développées. Dans l'anime, les ellipses établies au niveau de la gouttière disparaissent ${ }^{15}$. Les réalisateurs ajoutent des actions ou des plans sur d'autres personnages afin de les combler.

5 Étendre les séquences narratives du manga n'étant pas suffisant pour tenir l'écart de diffusion entre bande dessinée et anime, les scénaristes exploitent les ellipses du manga pour fabriquer des récits inédits, des fictions transfuges mettant en scène les personnages secondaires. Relativement rares dans la littérature, ces interpolations transfictionnelles (Saint-Gelais $2011: 84$ ) sont très répandues dans le media mix japonais car elles permettent aux scénaristes de créer des événements inédits à la manière de l'auteur. Il ne s'agit pas à proprement parler de pastiches dans la mesure où il est question d'adaptations sur un autre média. Mais si l'on prend cette notion au sens large, en tant qu' 'imitation des qualités ou des défauts propres à un auteur ou à un ensemble d'écrits» (Aron 2008: 5), il semble que les équipes de Toei aient cherché à reproduire sur un autre support le style de Toriyama. Ces interpolations reprenant des situations déjà apparues dans la bande dessinée, elles engendrent une sérialité qui renforce celle qui était déjà mise en œuvre dans le manga.

6 L'anime duplique les séquences narratives et les amplifie par des interpolations afin de maintenir la chronologie des médias. Pour le téléspectateur n'ayant pas lu le chapitre du manga correspondant plusieurs mois auparavant, la tension narrative est produite par le suspense (Baroni 2007). Pour les lecteurs regardant la série, la tension narrative repose plutôt sur la surprise (ibid.). Les incises déjouent la prévisibilité de l'histoire et accréditent la possibilité d'aller au-delà du récit exposé dans le manga. Elles postulent l'existence d'un «monde fictif autonome, puisque indéfiniment explorable au-delà de ce qu'énonçait le récit initial» (Saint-Gelais $2011: 89$ ).

7 Parallèlement à la diffusion de la série animée, un long-métrage est produit chaque année mais le scénario ne reprend que rarement le récit du manga. Généralement, ce film constitue une longue interpolation transfictionnelle pastichant l'œuvre de Toriyama et permettant aux lecteurs du manga comme aux téléspectateurs de la série télévisée de profiter d'une tension narrative liée au suspense procuré par une intrigue inédite. Dans un grand nombre de cas, il existe toutefois des incohérences mineures ${ }^{16}$. Ces films peuvent également constituer des versions contrefictionnelles (Saint-Gelais 2011), notion qui correspond à des récits modifiant le cours de l'histoire tel qu'il était établi jusque-là et qui réfèrent à des mondes possibles distincts ${ }^{17}$. Les adaptations audiovisuelles produisent ainsi des expansions venant compléter le manga (cas de la série) et des versions le contredisant (cas des films). 


\section{Un récit réticulaire}

8 La création parallèle du manga et de l'anime engendre des rapports d'imitation (reprise des événements et utilisation des planches du manga comme story-board ${ }^{18}$ ), de complétude (cas des incises transfictionnelles) mais aussi de concurrence. En effet, le feuilleton télévisé puise dans la même matrice sérielle que l'œuvre originale et oblige l'auteur à modifier son récit pour innover. Elle entre en concurrence avec le manga dans l'exploitation des scénarios possibles à partir d'un même matériau puisque les péripéties actualisées dans l'anime ne peuvent pas être reprises dans le manga. Par le biais des interpolations, les épisodes tendent à actualiser toutes les combinaisons possibles et non exploitées des topos dans le manga. Dans le volume 17, les personnages évoquent l'ascendance extraterrestre du héros qui appartient à l'espèce des saiyans. Les scénaristes élaborent alors un preque ${ }^{19}$ (Saint-Gelais 2011: 77) permettant d'étoffer l'univers fictif en mettant en scène Bardock, père de Son Goku, dans un épisode spécial (17 octobre 1990). À cette occasion, l'anime est en quelque sorte en «avance » sur le manga puisqu'il dévoile des personnages qui n'ont pas été montrés jusque-là, ce qui va à l'encontre de la chronologie des médias. Toriyama n'intervient jamais directement dans le processus d'adaptation mais il corrige et valide les caractéristiques graphiques des nouveaux personnages apparaissant dans l'anime. L'auteur est en quelque sorte obligé de trouver un moyen de justifier pourquoi il n'a pas mis en image le père de Son Goku et il met pour cela au point une astuce : chez ces extraterrestres, les pères et les fils ont le même visage. Cette solution est acceptable pour le lecteur et le téléspectateur naïf car elle légitime l'ellipse dans le manga et conforte l'idée que ces personnages ne sont pas humains. Dans ce cas précis, l'adaptation a infléchi l'œuvre originale sur un matériau de l'univers diégétique. Par ailleurs, dans le cas des épisodes filler 108 à 117, la série propose une sequel aux événements du quatrième film (DBZ : À la poursuite de Garlic, 1989), signe que l'univers fictif s'étend à travers plusieurs supports et non simplement dans le livre même si le récit de Toriyama est la source principale ${ }^{20}$.

$\mathrm{Au}$ niveau graphique, l'auteur a progressivement changé son style pour intégrer les lignes droites et les formes plus en pointe de l'anime afin de refléter la violence des combats. Il avait en effet remarqué que les dessinateurs de l'anime adoptaient un style plus anguleux. Durant les dix années d'élaboration en parallèle de la version papier et audiovisuelle, la série ne s'est donc pas contentée d'être une simple reprise du récit en bande dessinée. Elle a poussé le créateur original à modifier ses récits et son esthétique. Dans le cas de $D B$ il n'y a donc pas une simple adaptation d'un support à un autre mais une influence croisée puisque le manga doit sans cesse se repositionner par rapport à l' anime. Les fictions élaborées dans un contexte industriel très contraignant n'empêchent donc pas les artistes de s'exprimer et de s'influencer réciproquement. Il n'y a ainsi plus de subordination hiérarchique entre un support et un autre mais un réseau rhizomatique de relations plus horizontales.

10 La différence ne réside pas seulement dans la médiagénie (Marion 1997) des différents supports qui impliquerait des transformations spécifiques à un média donné, mais dans le moment de création du monde fictif et la nature de l'œuvre source. Par ailleurs, dans le cas d'une licence comme $D B$, la longévité de l'exploitation est un facteur important dans l'évolution de l'univers diégétique. Ainsi, la perte d'influence progressive des éditeurs de manga au profit des industries audiovisuelles et vidéoludiques au cours des 
années 2000 explique pourquoi le récit se déploie aujourd'hui dans les films et les jeux vidéo avant d'être repris et modifié dans les séries télévisées et les mangas.

\section{Au livre comme cible}

\section{Perte d'influence du livre}

11 Parallèlement au circuit d'adaptation partant du livre vers l'audiovisuel et le vidéoludique, d'autres possibilités ont été exploitées. À partir du milieu des années 1990, ces variantes du dispositif initial ont pris de l'ampleur. Cette période correspond à une perte d'influence des éditeurs papier face aux autres secteurs économiques comme le jeu vidéo. En effet, après la fin de $D B$, les ventes du Shônen Jump ont baissé sans jamais retrouver leur niveau de 1995. Les éditeurs ont alors commencé à adapter plus régulièrement des licences populaires issues du jeu vidéo comme Pokémon afin de recruter le public du jeu vidéo, le changement de média source manifestant le bouleversement de l'équilibre dans l'écosystème médiatique ${ }^{21}$. Depuis la fin des années 1990, la multiplication des dispositifs de media mix non basés sur un manga entraîne une évolution dans la création de ces fictions industrielles.

L'essai Tezuka est mort de Go Ito (2005) témoigne de la prise en compte de cette modification du media mix. L'auteur y rappelle que le manga n'est plus le premier espace de rencontre avec la fiction : « Nowadays, it has become common for children to experience anime and videogames before manga, and oftentimes discover manga only later (in some cases postpuberty) ${ }^{22}$ " (Ito 2011: 78). Comme le remarque Hutcheon (2013 : XIV), les industries culturelles liées au jeu vidéo ont eu tendance à adapter davantage un monde fictif qu'un récit. Dans le cas du transmedia storytelling, cette évolution favorise la cohérence d'un univers unique. Dans le media mix japonais, cela accroît les versions contrefictionnelles d'événements se déroulant dans un même univers ou, si l'on préfère, la prolifération d'univers parallèles incompatibles ${ }^{23}$.

\section{Développement narratif inédit sous forme animée}

Durant la première phase d'exploitation de $D B$, le récit principal se déployait sur le support papier, les autres supports proposant des traductions intersémiotiques, des interpolations, des variantes, des prequels. Par la suite, la fiction narrative se structure au niveau des anime et des jeux vidéo, la bande dessinée devenant une adaptation de ceux-ci.

Seque ${ }^{24}$ se déroulant dix ans après les événements de $D B$, la série animée $D B G T$ (1996-1997, 64 épisodes) permet d'exploiter le succès d'une œuvre achevée, en la faisant rebondir vers de nouvelles péripéties. Cette continuation allographe de l'œuvre de Toriyama est ensuite déclinée en deux livres décrivant l'univers de manière encyclopédique $^{25}$, des jeux vidéo ${ }^{26}$, un anime comics (2013), c'est-à-dire une bande dessinée formée à partir des photogrammes de la série animée. Il s'agit d'une simple traduction intersémiotique du support animé sans ajout d'élément diégétique nouveau par rapport à l'anime ${ }^{27}$. Ainsi, le livre est devenu le média cible dans un circuit d'adaptation où la fiction se développe dans la série télévisée. Fausse nouveauté narrative mais vraie création sérielle, l'anime comics permet d'occuper le terrain médiatique en attendant la production d'une séquence inédite sur un autre support. 
15 L'univers fictif a continué de croître par le biais des films proposant des incises transfictionnelles, des versions (contrefictionnelles ou non) et des sequels. Les films supervisés par Toriyama (DBZ: Battle of Gods en 2013 et DBZ: La Résurrection de 'F' en 2015) constituent la suite du manga tandis que la série télévisée DB Super (en cours depuis juillet 2015) propose une version différente et amplifiée de ces mêmes événements ${ }^{28}$. Films et série constituent ainsi deux versions similaires mais se déroulant dans des univers parallèles incompatibles. La nouvelle série télévisée est actuellement adaptée sous la forme d'un manga portant le même titre et dessiné par Toyotarô sous la supervision d'Akira Toriyama.

16 Ces versions animées divergentes s'ajoutent au fait que dans la diégèse initiale, le personnage de Trunks remonte le cours du temps et engendre plusieurs futurs mis en scène dans la série animée et dix univers parallèles dont l'existence est mentionnée dans le dix-huitième film. Cette prolifération de versions et de mondes est difficilement contenue puisqu'à mesure que la série DB Super progresse les personnages évoluent dans différents univers. Ainsi, la multiplication des récits contrefictionnels est en quelque sorte thématisée au niveau diégétique par les univers et futurs alternatifs sans que cela engendre une hiérarchie stricte entre les versions qui seraient canoniques et celles qui ne le sont pas.

\section{Autres versions et autres publics en livre}

17 Parallèlement à l'élaboration de nouveaux récits audiovisuels se déroulant dans le même univers, l'éditeur Shueisha a cherché à poursuivre l'exploitation de l'intrigue initiale après la fin de la publication du manga en proposant différentes versions de ce récit. À la fin de la diffusion du dessin animé à la télévision en 1996, l'engouement pour la série est resté suffisant pour vendre à la fois les volumes du manga et les encyclopédies liées à la licence ${ }^{29}$. Afin de prolonger l'intérêt pour cet univers fictionnel, les 42 volumes originaux ont ensuite été republiés dans un format différent : DB Perfect Edition (2002-2004, 34 volumes). Puis la maison d'édition a produit une adaptation de la série animée en anime comics (2005-2010, 39 volumes) $)^{30}$.

Dans cette sorte d'adaptation à rebours, le créateur de l'anime comics enlève non seulement les fillers mais aussi les plans inutiles pour essayer de retrouver la mise en page originale du manga. Cela permet d'une part de garder une relative fidélité, et d'autre part de gagner du temps dans la sélection des images à garder par rapport aux milliers de dessins de l'anime. Dans le passage de l'audiovisuel au livre, il n'y a pas d'ajout ou de création de nouvelles images puisque l'anime comics est réalisé à partir de captures d'écran ou des celluloïds ${ }^{31}$ issus de la réalisation du dessin animé. La taille de ces dessins conditionne donc celle de la case au sein de la planche. Ce processus d'adaptation ne génère pas de nouveau contenu narratif, mais contribue à densifier des éléments diégétiques à travers la transposition de certains épisodes filler (récits inédits créés pour l'anime qui sont ici préservés sur un support papier). La conservation de certaines séquences narratives témoigne de leur popularité auprès du public et contribue à leur fixation dans les mémoires des amateurs et dans le monde fictif.

À mesure que la fiction narrative s'étend en colonisant de nouveaux supports, elle continue de se déployer sur papier sous la forme d'adaptations du récit initial à un public plus jeune. Dans la série DB SD (2010), l'histoire originale est condensée et le style de dessin modifié pour être plus attrayant auprès d'un lectorat cible différent ${ }^{32}$. 
L'intrigue est une réécriture dans laquelle l'univers est présenté de manière plus cohérente qu'il n'a été initialement créé. $D B$ étant un feuilleton, Toriyama ne prévoit pas toujours les rebondissements de l'histoire et l'origine extraterrestre de Son Goku n'est par exemple dévoilée qu'après dix-sept volumes tandis que dans $D B S D$, les extraterrestres apparaissent dès le premier chapitre. Pour le lecteur connaissant déjà la licence, il s'agit d'un clin d'œil humoristique alors que pour celui qui la découvre, la référence fonctionne comme une forme de prolepse.

En France, Hachette publie depuis 2010 des novellisations du manga spécifiques au marché françaiis ${ }^{33}$. Éditées dans la collection "Bibliothèque verte ${ }^{34}$ ", elles sont destinées à un public de 8-10 ans et correspondent à la stratégie actuelle de l'éditeur qui oriente cette collection vers l'adaptation en romans de films et de séries télévisées ${ }^{35}$. Que le livre soit devenu le média cible de l'adaptation manifeste la domination du secteur audiovisuel en matière de création d'univers fictionnels. Dans les deux cas, il s'agit des mêmes histoires mises en récit à destination d'un public plus jeune. Chaque nouvel opus réactive l'ensemble des récits déjà produits et contribue à faire du manga initial un élément parmi d'autres dans un réseau fictionnel toujours en croissance.

21 Après avoir été des adaptations avec des variantes minimes, les séries animées sont devenues les lieux d'expansion de l'univers fictif, les continuations se matérialisant d'abord sous la forme audiovisuelle avant d'être reprises en format papier. Les adaptations en livre (anime comics, manga, novellisation) n'apportent aucune nouveauté au monde fictif mais leur parution permet à la licence de conserver une bonne couverture médiatique et de continuer de capter l'attention des amateurs. À partir des années 2010, parallèlement aux continuations animées, le jeu vidéo prend également en charge des formes d'enrichissement de la diégèse.

\section{Le livre comme possible}

\section{Du support marketing à l'expansion narrative}

Après l'explosion de la bulle économique du secteur de l'animation ${ }^{36}$ les studios de production ont vu leurs conditions de travail et leur situation financière se dégrader pour aboutir à une série de faillites en $2010^{37}$. Depuis la crise économique mondiale de 2008, les agences de communication et les chaînes télévisées faisant partie des comités de production des anime sont plus frileuses à l'idée d'investir dans des projets innovants dont le succès n'est pas garanti. Ce climat favorise les suites et les reboot de séries $^{38}$ mais oblige les différents acteurs économiques à renforcer leur collaboration en vue de promouvoir une même licence. Dans ce contexte, de nouveaux circuits d'adaptation voient le jour et utilisent le manga en presse comme un maillon dans une campagne globale de communication. C'est notamment le cas pour les jeux vidéo $D B$ qui, depuis 2010, deviennent des plateformes de développement de l'intrigue. Auparavant, ceux-ci se contentaient de reprendre des portions du récit de l'anime ou du manga. Les joueurs incarnaient soit Son Goku soit l'un de ses compagnons dans des jeux d'action-aventure ou des jeux de combat. Ils ne pouvaient que reprendre un personnage existant alors qu'à partir des titres de 2010, ils peuvent désormais créer un nouveau personnage dans l'univers fictif. 

collectionner, les utilisateurs incarnent des personnages du monde de $D B$ dans un jeu de combat ${ }^{39}$. Afin de justifier l'existence d'une transformation chez le personnage de Bardock qui n'existe pas dans le manga de Toriyama mais qui avait été créé pour un prequel disponible sous la forme d'un épisode télévisé, les ayants droit ont ajouté une histoire à l'univers fictif. Les événements de l'épisode spécial DBZ : Baddack contre Freezer (1990) ont bénéficié d'une expansion narrative sous la forme de trois chapitres dessinés par Naho Ôshi publiés dans le magazine V Jump durant l'été 2011 (DB: Episode of Bardock) qui a ensuite été adaptée en un épisode télévisé spécial en décembre 2011. Outre le fait que le manga poursuit le récit commencé dans un épisode télévisé, il participe à la promotion d'un nouvel épisode animé et il permet d'expliquer un élément du jeu d'arcade DB Heroes. Le titre appartenant au genre du jeu de combat, l'aspect narratif est relégué sur d'autres supports (papier et audiovisuel) servant à justifier les nouveaux éléments implémentés en jeu au fil de l'exploitation du titre en salles d'arcade. Autrement dit, le jeu vidéo devient le secteur où s'élabore la fiction même si le manga reste le média où le récit se déploie.

De la même manière, les séries DB Heroes: Victory Mission ${ }^{40}$ et DB Heroes: Charisma Mission ${ }^{41}$ servent à la fois de support promotionnel pour le jeu vidéo et de tutoriel pour expliquer aux lecteurs comment jouer en représentant à la fois les cartes à collectionner, les bornes d'arcade et les combats. Ces mangas opèrent une remédiation du jeu vidéo en adaptant le support médiatique, en reprenant les cartes et les règles du jeu dans la fiction narrative ${ }^{42}$. Ce dispositif se justifie par l'importance de la couverture médiatique qu'il apporte et le lien qu'il crée entre les lecteurs et les potentiels joueurs qu'elle permet de recruter.

Les intrigues de ces mangas servent de toile de fond pour les jeux. Dans le cas de $D B$ Heroes: Charisma Mission et sa suite, la série met en scène trois personnages qui s'occupent des bornes d'arcade et du bon fonctionnement du jeu. Une même forme de complémentarité se retrouve dans le cas de DB Fusion (jeu de rôle sur Nintendo 3DS, 2016) et le manga du même titre publié depuis mai 2016 dans Saikyō Jump ${ }^{43}$. Le transmedia storytelling semble donc surtout servir à promouvoir les mécaniques du jeu et à lui donner un arrière-plan fictif. Au lieu d'être un événement, au sens où il s'agit d'une pratique ritualisée par la périodisation de la diffusion d'un élément diégétique inédit, la sortie d'un nouveau chapitre de manga sert avant tout à préparer l'arrivée sur le marché d'un autre média. Elle assure une couverture médiatique en amont de la sortie du jeu vidéo.

Si les chapitres de DB Heroes ne comportaient primitivement que quelques planches présentant des joueurs en action, ils s'étendent à partir de 2014 sur une quinzaine de pages permettant le développement d'une intrigue se déroulant dans l'univers du jeu vidéo. Les héros y sont des joueurs qui, en utilisant les bornes d'arcade DB Heroes, retournent dans le passé afin de revivre les combats sans que cela perturbe le cours des événements ${ }^{44}$. Mais des démons altèrent le cours des sessions de jeu et les joueurs doivent les combattre pour rétablir le déroulement normal de l'histoire. Ces chapitres ajoutent des éléments à l'univers fictif tout en promouvant de nouvelles mécaniques de jeu implémentées peu avant dans les bornes d'arcade (nouvelles cartes, nouvelles compétences et nouveaux personnages). Par ailleurs, l'intrigue reprend des situations déjà apparues dans le manga ou l'anime, en proposant des variantes sérielles à partir de la même matrice ${ }^{45}$. Au lieu d'être un simple support de communication, DB Heroes: 
Victory Mission évolue donc pour devenir une continuation narrative de la série animée $D B$ GT. C'est une exception remarquable par rapport à d'autres bandes dessinées dérivées de l'univers $D B$ qui n'atteignent pas cette indépendance par rapport au jeu dont ils font la promotion. Néanmoins, après la prépublication en magazine, cette série n'a pas bénéficié d'une republication en un volume papier, les chapitres n'étant regroupés que sur le site web de l'éditeur ${ }^{46}$, comme si le livre devenait un possible non actualisé de la bande dessinée.

\section{Le jeu vidéo comme continuation métaleptique}

Parallèlement à $D B$ Heroes, un MMORPG (jeu de rôle en ligne massivement multijoueur) est sorti en 2010 au Japon et en Corée du Sud ${ }^{47}$. Les joueurs peuvent y élaborer un avatar à partir des espèces vivant dans l'univers de $D B$ et participer à des tournois d'arts martiaux. Un système de quêtes leur permet de voyager dans le temps et de revivre des portions de l'intrigue du manga ou de l'anime. La série de jeux sur console DB Xenoverse (2015 et 2016) adapte les quêtes du MMORPG qui servent alors de base pour l'intrigue inédite. Afin de rétablir la chronologie et le déroulement de l'histoire telle qu'elle est relatée dans les livres et anime précédents, le joueur doit aider le personnage de Trunks à retourner dans le passé et combattre des démons. Au lieu d'être la simple reprise interactive de la fiction, ce jeu vidéo propose une sorte de réécriture de l'histoire connue jusqu'alors. Il propose par exemple de corriger une version contrefictionnelle du récit tel qu'il apparaît dans le manga et poursuivi dans le dix-huitième film de la licence. Le joueur doit intervenir pour que les événements clés se déroulent comme dans l'histoire initiale et, ce faisant, il crée une autre version, une continuation métaleptique où la diégèse initiale est annexée dans une fiction englobante située à un autre niveau. Parallèlement au développement de la fiction par le biais de la série télévisée $D B$ Super, le jeu vidéo propose ainsi une réécriture de la diégèse des œuvres précédentes dans une métafiction incluant potentiellement les extensions narratives à venir sur d'autres supports.

Dans le jeu, les protagonistes ne sont pas explicitement désignés comme des personnages de mangas ou de films. Néanmoins, les perturbations dans la fiction originale sont représentées en jeu par une remédiation du manga sous la forme de volumens ${ }^{48}$. Lorsque la déesse du temps les déroule, les images du passé sont projetées sur la feuille comme sur un écran télévisé. Ces rouleaux symbolisent donc à la fois les récits en bande dessinée et en animation que le jeu vidéo permet de modifier. Lorsque le joueur intervient dans ces séquences narratives modifiées, il prend le volumen concerné en main et semble disparaitre pour entrer à l'intérieur de celui-ci. Par ailleurs, les phases de jeu sont entrecoupées par des cinématiques ${ }^{49}$ (ou cut scenes) réalisées soit en trois dimensions avec le moteur graphique du jeu soit en deux dimensions sous forme d'animation ${ }^{50}$. Ces séquences où le joueur regarde l'action se dérouler au lieu d'interagir forment comme des condensés de films insérés dans le jeu. Lorsqu'elles sont mises bout à bout (en excluant les phases de jeu) elles durent un peu plus d'une heure et demie et contiennent le matériau narratif inédit ajouté à l'univers de $D B$. Ainsi, le livre n'est plus le média source de la fiction narrative mais il reste visible à travers le volumen comme l'un des supports de la diégèse. remédiations des jeux vidéo dans le manga (DB Heroes) ou des supports papier et 
audiovisuel dans le jeu vidéo (DB Xenoverse). Le livre n'est plus la source de la fiction ou un passage obligé dans le processus d'adaptation, ce qui témoigne de son statut actuel : objet menacé de disparition face à l'essor du livre numérique. Le jeu vidéo diégétise en quelque sorte la situation de l'édition papier. En effet, le secteur de l'édition papier devenant de moins en moins rentable pour les éditeurs japonais, ceux-ci tentent de trouver des leviers de croissance dans le développement du manga numérique ${ }^{51}$. Toutefois, aucun modèle économique viable n'est encore disponible, ce qui laisse au format matériel du temps pour évoluer.

\section{Conclusion}

Dispositif de maximisation de la visibilité médiatique, le media mix permet à une même fiction d'être présente sur de multiples supports tout en assurant une importante rentabilité pour tous les ayants droit. Les récits produits ne sont pas liés par une simple hiérarchie d'œuvre source et de produits dérivés. Ils forment des univers diégétiques parallèles divergents témoignant des fertilisations croisées des imaginaires des créateurs. Les reprises avec traduction intersémiotique se mélangent avec les incises, les prequels, les versions contrefictionnelles et les continuations métaleptiques. Le livre $\mathrm{y}$ est à la fois central (origine du monde fictif) et accessoire (adaptation des anime, novélisation), son rapport à la création de nouveaux éléments narratifs étant lié à sa place dans l'écosystème médiatique japonais. Ce développement réticulaire met à mal la notion d'auteur telle qu'elle apparaît dans la littérature moderne en tant qu'origine de l'œuvre, individu ayant une autorité sur le texte (auctorialité) ${ }^{52}$, «principe par lequel on entrave la libre circulation, la libre manipulation, la libre composition, décomposition, recomposition de la fiction » (Foucault $2001: 839$ ). Dans le cas des récits produits au sein du media mix, la circulation transmédiatique et l'expansion diégétique sont des œuvres collectives impliquant de nombreux individus, ce qui entraîne un régime d'auctorialité différent favorisant la prolifération de la fiction. Ce dispositif d'adaptation met en lumière le caractère collectif de la création qui avait été dissimulé dans la création de la réputation de l'artiste dans les mondes de l'art qui « ignorent systématiquement tout ce que cette production doit à l'action d'autres participants " (Becker [1982] 2010 : 357).

\section{BIBLIOGRAPHIE}

Aron, Paul, 2008, Histoire du pastiche, Paris, PUF.

Baetens, Jan, 1991, « Pour une poétique de la gouttière », Word \& Image, vol. 7, n 4, p. 365-376.

Baroni, Raphaël, 2007, La Tension narrative : suspense, curiosité et surprise, Paris, Seuil.

Baudry, Julien et Litaudon, Marie-Pierre, 2016, « Hachette entre héritage et renouvellement

(1920-1960) : comment "faire collection" face au défi des albums "transmédiatiques" ?", Strence, 
[En ligne], http://strenae.revues.org/1631.

DOI : $10.4000 /$ strenae. 1631

Becker, Howard, [1982] 2010, Les Mondes de l'art, Paris, Flammarion.

Berndt, Jacqueline, 2008, « Considering Manga Discourse: Location, Ambiguity, Historicity », dans M. Wheeler Macwilliams (dir.), Japanese Visual Culture: Explorations in the World of Manga and Anime, Armonk, M.E. Sharpe, p. 295-310.

Besson, Anne, 2015, Constellations : des mondes fictionnels dans l'imaginaire contemporain, Paris, CNRS Éditions.

Blanchet, Alexis, 2010, Des pixels à Hollywood. Cinéma et jeu vidéo, une histoire économique et culturelle, Châtillon, Pix'n Love.

Bolter, Jay David et Grusin, Richard, 1999, Remediation: Understanding New Media, Cambridge, MA, MIT Press.

Bortolotti, Gary R. et Hutcheon, Linda, 2007, « On the Origin of Adaptations: Rethinking Fidelity Discourse and "Success"-Biologically », New Literary History, n 38, p. 443-458.

Bouissou, Jean-Marie, 2012, Manga : histoire et univers de la bande dessinée japonaise, Arles, P. Picquier.

Compagnon, Antoine, s. d., "Quatrième leçon : Généalogie de l'autorité », dans Qu'est-ce qu'un auteur?, [Cours en ligne], http://www.fabula.org/compagnon/auteur4.php.

Foucault, Michel, 2001, «Qu'est-ce qu'un auteur ? », dans Dits et écrits : 1954-1975, Paris, Gallimard, p. 817-849.

Hutcheon, Linda, [2006] 2013, A Theory of Adaptation, Londres, Routledge, $2^{\mathrm{e}}$ édition.

Ito, Go, 2005, Tezuka izu deddo: hirakareta manga hyôgenron e, Tôkyô, NTT Shuppan.

Ito, Go, 2011, « Tezuka is Dead: Manga in Transformation and its Dysfunctional Discourse », Mechademia, $\mathrm{n}^{\circ}$ 6, p. 69-82.

Jenkins, Henry, 2006, Convergence Culture, New York, New York University Press.

Johnson, Derek, 2013, Media Franchising: Creative License and Collaboration in the Culture Industries, New York, New York University Press.

Koyama-Richard, Brigitte, 2007, Mille ans de manga, Paris, Flammarion.

Marion, Philippe, 1997, « Narratologie médiatique et médiagénie des récits », Recherches en communication, $\mathrm{n}^{\circ} 7, \mathrm{p} .61-88$.

Richard, Olivier, 2011, Akira Toriyama, Le Maître du manga, Paris, Éditions 12bis.

Saint-Gelais, Richard, 2011, Fictions transfuges, la transfictionnalité et ses enjeux, Paris, Seuil.

Steinberg, Marc, 2011, « Condensing the Media Mix: Multiple Possible Worlds in the Tatami Galaxy ", Canadian Journal of Film Studies, vol. 21, nº 2, p. 71-92.

Steinberg, Marc, 2012, Anime's Media Mix: Franchising Toys and Characters in Japan, Minneapolis, University of Minnesota Press.

Thompson, Kristin, 1980, «Implications of the Cel Animation Technique », dans The Cinematic Apparatus, Londres, Palgrave Macmillan, p. 106-120.

Toriyama, Akira, 1999, Le Dictionnaire de DB, Grenoble, Glénat. 


\section{NOTES}

1. Les termes "licence» et "franchise » sont issus du vocabulaire du droit de la propriété intellectuelle et désignent des contrats d'exploitation d'une œuvre. Par extension, ils correspondent aux fictions elles-mêmes dans les discours médiatiques, marketing, mais aussi des fans. Johnson (1997) étudie les franchises américaines et montre que la société détenant temporairement les droits d'exploitation peut choisir d'élaborer son univers fictif sans tenir compte des précédentes réalisations. Dans le cas de la franchise Marvel, plusieurs versions de Spiderman cohabitent au cinéma selon les producteurs. The Amazing Spiderman (2014) se déroule ainsi dans un univers différent du film Captain America: Civil War (2016). Le terme licence est plus utilisé dans le domaine des productions japonaises et apparaît régulièrement dans les communiqués de presse des éditeurs (voir par exemple: http://www.toei-animation.com/fr/ content/nouvel-agent-pour-dragon-ball-en-Allemagne).

2. La plupart des chercheurs analysent un média et évoquent rapidement les adaptations au lieu d'étudier les processus transmédiatiques au sein d'une licence (Bouissou 2012 et Koyama-Richard 2007).

3. Connu sous le titre Astro, le petit robot en France, cette licence créée par Osamu Tezuka en manga dès 1952 a été adaptée en série télévisée en 1963. Pour Steinberg (2012), cette œuvre a institué le système du media mix, c'est-à-dire un environnement médiatique où le récit est adapté à la télévision par le biais d'un partenariat avec une entreprise tierce utilisant les personnages comme élément promotionnel (Astro figurant sur des autocollants dans des boites de chocolats).

4. Pour une analyse du media mix, voir Steinberg (2012) qui étudie les formes d'adaptation chez l'éditeur Kadokawa.

5. Ce dernier cas correspond au transmedia storytelling défini comme « un processus à travers lequel les éléments d'une fiction sont dispersés sur plusieurs plateformes médiatiques dans le but de créer une expérience de divertissement coordonnée et unifiée » (Jenkins 2006 : 95-96).

6. En l'occurrence, nous ne nous intéresserons qu'aux interactions entre les secteurs du livre, de l'audiovisuel et du jeu vidéo au Japon entre 1984 et 2016. Nous n'aborderons pas la question des circulations transnationales car elles n'ont pas d'impact sur la création de l'univers diégétique pensé uniquement pour un public japonais, contrairement aux productions cinématographiques hollywoodiennes conçues dès le départ pour une diffusion mondiale. D'ailleurs les éditeurs japonais n'ont établi des filiales européennes qu'à partir du début des années 2000, signe que le marché occidental a peu de poids par rapport au marché interne.

7. Le succès en termes d'adaptation est défini par Gary Bortolotti et Linda Hutcheon par les trois critères suivants : «persistence, abundance, and diversity" (2007 : 452).

8. En 2015, à partir du manga initial d'Akira Toriyama (1984-1995), ont été produit cinq séries de manga, deux séries d'anime comics (adaptation des récits audiovisuels en bandes dessinées utilisant des captures d'écran pour former les cases), cinq séries d'animation, dix-neuf longs métrages d'animation, quatre téléfilms d'animation, cinq OAV (longs métrages destinés au marché de la vidéo domestique), trois films live dont deux non officiels, et une centaine de jeux vidéo disponibles sur diverses plateformes. Les 42 volumes du manga initial de Toriyama se sont écoulés à travers le monde à plus de 240 millions d'exemplaires dont 17 millions en France.

9. Actuellement, le magazine n'est diffusé qu'autour de 2,5 millions d'exemplaires par semaine.

10. Il faudrait aussi inclure les chaînes de télévision, les agences de communication et l'éditeur des musiques qui investissent également dans la création de la série d'animation, mais pour la clarté du propos, nous nous limitons aux trois pôles Shueisha, Toei et Namco-Bandai. Les mêmes acteurs économiques sont impliqués dans la production de One Piece.

11. Abréviation de l'anglais animation, le terme est employé par les Japonais pour désigner les séries télévisées souvent en animation limitée. Il s'oppose à manga eiga (littéralement «film manga ») terme employé pour les longs métrages, notamment ceux de Hayao Miyazaki. 
L'opposition entre les deux termes repose à la fois sur une différence de circuit de production et de diffusion mais aussi sur des techniques d'animation distinctes. Le terme générique français (dessin animé) ne rend pas compte de cette nuance.

12. Le manga Dragon Ball (1984-1995) a été adapté en deux séries: Dragon Ball (1986-1989, 153 épisodes correspondant aux volumes 1 à 17) et Dragon Ball Z (1989-1996, 291 épisodes correspondant aux volumes 18 à 42 ).

13. Le terme filler vient de l'anglais to fill, « remplir ». Ainsi, les épisodes 108 à 117 de DBZ sont des fillers s'insérant entre deux épisodes correspondant au chapitre 329.

14. Par ailleurs, une durée de vingt-cinq minutes permet d'insérer une coupure publicitaire après le générique et au milieu de l'épisode, alors qu'un format plus court rend cette interruption moins efficace. Lorsque les anime sont plus courts, ils sont généralement regroupés dans une série anthologique plus longue. Ainsi Oruchuban Ebichu d'une durée de 9 minutes a été diffusé dans le cadre du programme Anime Ai no Awa hour (1999) qui comportait d'autres séries courtes.

15. Le terme gouttière fait référence à l'espace blanc entre les cases et à l'ellipse temporelle permettant au lecteur d'imaginer la scène (Baetens 1991).

16. Par exemple, dans le quatrième film, DBZ : À la poursuite de Garlic (juillet 1989), Son Gohan (fils du héros Son Goku) est connu de tous les personnages alors que l'intrigue se déroule avant les événements décrits dans la série DBZ (à partir d'avril 1989) où Son Goku présente son fils à ses amis.

17. C'est le cas du premier et du troisième film de la licence : DB : La Légende de Shenron (1986) et DB : L'Aventure mystique (1988).

18. Dans une interview, Shigeyasu Yamauchi, l'un des réalisateurs de la série Dragon Ball $Z$ revient sur le processus d'adaptation: "Je me souviens qu'une fois j'avais juste une petite page de dialogues entre Goku et Végéta qui discutaient au-dessus de la Terre. C'est tout ce que j'avais pour faire un épisode! Je me suis débrouillé en inventant des scènes de dialogues entre les personnages qui étaient restés sur Terre » (cité dans Richard $2011: 104$ ).

19. Le terme désigne les continuations narratives dont l'histoire précède celle d'une œuvre produite antérieurement.

20. Dans le cas de ces épisodes fillers, le récit adopte la méthode du transmedia storytelling dans le sens où le récit relate la suite d'événements décrits sur un autre support, série et film entrant dans une relation de complémentarité. Il faut donc avoir vu le film pour comprendre ces épisodes alors que dans la plupart des cas, la série propose des incises transfictionnelles et donc des relations d'événements mineurs.

21. L'univers diégétique du jeu vidéo Pokémon de 1995 a été utilisé dans plusieurs séries de mangas (dont la première date de 1997), une série télévisée (toujours en cours depuis 1997), un jeu de cartes à collectionner (1999) et de multiples produits dont des parcs d'attraction. Initialement éditeur de jeux vidéo, Square Enix a créé des magazines de prépublication comme Monthly Shōnen Gangan (1991) et Monthly GFantasy (1993) dans lesquels les licences élaborées par la société sont adaptées en bandes dessinées : Final Fantasy, Dragon Quest, Kingdom Hearts, Star Ocean, etc.

22. «Il est devenu courant que les enfants pénètrent dans la fiction par le biais de l'anime ou du jeu vidéo avant de lire le manga correspondant. Dans certains cas, ils ne le découvrent qu'à l'adolescence » (traduction personnelle).

23. Dans le cas de Fullmetal Alchemist, la série de 2003 et le premier film (2005) mettent en scène des personnages et un scénario différents du manga (2001-2010) formant ainsi une version contrefictionnelle, tandis que les six romans (2003-2007) proposent soit des adaptations reprenant les événements relatés sur un autre support soit des incises transfictionnelles de la série. En 2009, la seconde série télévisée reprend les événements décrits dans le manga.

24. Continuation narrative dont l'histoire se déroule après les événements décrits dans une œuvre produite antérieurement (Saint-Gelais 2011). 
25. DB GT Perfect Files, 1997.

26. DB: Final Bout en 1997 et DB GT: Transformation en 2005.

27. Cette forme de bandes dessinées réalisées à partir des photogrammes de la série n'est pas nouvelle. Les anime qui ne sont pas issus de mangas sont régulièrement adaptés sur un support papier de cette manière. La série télévisée Heidi (1974) réalisée par Isao Takahata (co-fondateur du studio Ghibli) a ainsi été l'objet d'un anime comics en 1996 édité par Tokuma Shoten.

28. Par exemple, au lieu de se situer dans un parc (film), l'anniversaire de Bulma se déroule sur un paquebot (série télévisée) et des incidents différents viennent émailler le récit qui suit le même schéma.

29. Il y a notamment eu un recueil d'illustrations et sept volumes intitulés DB Daizenshû qui n'ont pas tous été traduits en français.

30. Même s'ils ne suivent pas tout à fait la même chronologie que le manga ou la série, les films ont tous été adaptés en anime comics. Ainsi le premier film de la série DBZ (diffusé au cinéma le 15 juin 1989) est paru en anime comics le 24 septembre 1994. Cette date tardive s'explique par le fait que Toei continuait d'exploiter le film sous la forme de vidéos à domicile. Le film est en effet sorti en VHS et Laser Disc en février 1990.

31. Dans le dessin animé, un celluloïd est une feuille plastique transparente sur laquelle sont dessinées les images ensuite filmées pour produire un photogramme (Thompson 1980). Ces feuilles ont généralement la même dimension mais il y a parfois des celluloïd de taille irrégulière pour certains types d'effet de caméra.

32. La dessinatrice Naho Ôishi reprend le style de Toriyama dans une version en couleurs avec des personnages SD (abréviation de l'anglais Super Deformed), c'est-à-dire dessinés avec des proportions exagérées où la tête et le corps ont presque la même taille.

33. Contrairement à certaines séries basées sur un manga (Ghost in the Shell, Fullmetal Alchemist) dont la fiction a été ensuite développée sous la forme de romans ou de light novels (termes anglais utilisés par les Japonais pour désigner des romans illustrés destinés à un public de lycéens et d'étudiants), il n'existe pas d'adaptation de Dragon Ball en roman au Japon.

34. Hachette publie dans la même collection les novellisations de One Piece depuis 2011. L'éditeur est connu pour avoir adapté les films de Disney depuis 1934 (Baudry et Litaudon 2016). La collection Disney comporte plus de 600 titres (voir le site officiel http://www.hachette.com/en/ maison/hachette-jeunesse-collection-disney).

35. Cette politique éditoriale contraste avec les traductions de séries de romans jeunesse anglosaxons et les créations françaises qui étaient majoritaires durant les années 1950-1960.

36. À l'origine de cette bulle économique, il y a le succès colossal de la série Neon Genesis Evangelion (1995-1996) qui a permis de faire vendre une somme considérable de produits dérivés. Cela a incité une chaîne du satellite à se servir des anime comme produits d'appel. Diffusées gratuitement et à des horaires plus tardifs, les nouvelles séries produites visaient un public adulte ou de fans avec un pouvoir d'achat plus conséquent. Le succès de Cowboy Bebop (1998-1999) sur la chaîne Wowow a attiré d'autres investisseurs dans le secteur et a accru la production de séries plus originales mais aussi plus risquées.

37. En 2010, Group TAC, MadHouse et Future Planet ont déclaré faillite.

38. Lancée à l'occasion des vingt ans de la licence, Pretty Guardian Sailor Moon Crystal (2014) est une nouvelle série animée plus proche du manga original. De même pour les vingt ans de Dragon Ball, Toei Animation a lancé une version en HD de la série Dragon Ball Z sous le titre Dragon Ball Kai (2005-2015). Ghost in the Shell 2.0 (2008) est un remake du film de 1995 et les quatre moyens métrages Ghost in the Shell: Arise (2013-2014) forment un prequel à celui-ci.

39. Le placement des cartes sur la borne d'arcade permet de sélectionner les actions des personnages et remplace l'interface des manettes ou des joysticks.

40. Dessiné par Toyotarô dans le Saikyô Jump depuis 2012.

41. Dessiné par Yoshitaka Nagayama, dans le Saikyô Jump depuis 2013. 
42. La remédiation correspond à la représentation d'un support dans un autre média (Bolter et Grusin 1999), en l'occurrence ici les bornes d'arcade, les cartes et les interfaces du jeu vidéo qui se retrouvent dans les mangas.

43. Dessiné par Hiroshi Otoki, le titre ne comporte que trois chapitres introduisant les personnages de Pinich et Tekka (correspondant à l'avatar du joueur) qui sont transportés dans un univers parallèle où ils doivent recruter les guerriers de la licence $D B$ afin de combattre leur adversaire.

44. Dans l'univers diégétique, les bornes sont créées par Capsule Corporation, société fondée par le père de l'un des personnages principaux. Lorsque les joueurs commencent une partie, ils sont aspirés dans la borne d'arcade et peuvent interagir avec les guerriers du passé (sans que cela modifie le cours de l'histoire).

45. Ainsi, la réunification des sept boules de cristal ne permet pas d'invoquer un dragon magique exauçant les vœux mais un dragon malfaisant qui prend possession des joueurs et menace de détruire le monde en jeu (c'est-à-dire le passé où trois des joueurs enfants croisent leur grandpère) et le monde réel (où se déroulent les événements concernant les tournois d'arcade $D B$ Heroes).

46. Les vingt-huit chapitres sont désormais disponibles gratuitement en ligne sur le site de l'éditeur : http://vjump.shueisha.co.jp/push/dbh/.

47. Ce jeu n'existe plus depuis la fin 2013.

48. Le réalisateur Isao Takahata rapproche les volumens (ou emakimono en japonais) des mangas, notamment par le biais du Chôjû Giga, rouleaux où sont peints une caricature animale de la vie politique médiévale. Ce discours est erroné mais permet de donner une légitimité à ce média (Berndt 2008).

49. Ces séquences audiovisuelles préprogrammées se déclenchent lors de certaines phases de jeu et sont le principal lieu de développement narratif dans les jeux de combat (Blanchet 2010).

50. Il s'agit du même support que la série ou les films, même si le système de diffusion est ici un logiciel de jeu au lieu d'être un programme télévisé ou une salle de cinéma.

51. Les ventes de mangas numériques ont progressé de 42,8 millions de yens en 2009 à 65 millions en 2013.

52. Comme le rappelle Antoine Compagnon (s.d.), cette idée est néanmoins historiquement datée, l'auteur étant une catégorie inexistante durant l'Antiquité ou le Moyen Âge.

\section{RÉSUMÉS}

Dispositif de maximisation de la visibilité médiatique permettant à une même fiction d'être présente sur différents supports, le media mix a évolué au cours des trois dernières décennies en déplaçant le livre qui était à l'origine source des adaptations à la périphérie du circuit transmédiatique. Les récits produits ne sont pas liés par une simple hiérarchie entre une source et ses dérivés mais forment des univers diégétiques parallèles divergents où le livre est à la fois central et accessoire. Interpolations transfictionnelles, variantes contrefictionnelles et continuation métaleptique lient les différents formats (papier, audiovisuel, vidéoludique) où se développe successivement la fiction narrative. L'analyse de la licence Dragon Ball (1984-2016), exemplaire par sa longévité, la variété des déclinaisons transmédiatiques et son succès populaire, 
permet d'illustrer l'évolution de l'écosystème médiatique japonais et d'éclairer le fonctionnement d'autres univers fictifs (Naruto, One Piece).

Strategy for maximizing media exposure, the media mix allows one fiction to be present in different media while maintaining high profitability. Over the last three decades it has evolved by moving the book from the center to the periphery of the transmedia circuit. The stories are not linked by a hierarchy between a source and its derivatives. They form a network in which each narration interacts with the others: transfictional interpolations, conterfictional variants and metaleptic continuation bind different formats (paper, audiovisual, video game). In these diegetic universes which are divergent and parallel, books are both central (origin of the fictional world) and accessory (adaptation of anime, novelization). By its longevity, variety of transmedia variations and its popular success, the Dragon Ball license (1984-2016) illustrates the evolution of the Japanese media ecosystem and its analysis could contribute to understand how others fictional universes operate (Naruto, One Piece).

INDEX

Keywords : media mix, manga, transmédialité, anime, Dragon Ball, fiction, Japan, video games Mots-clés : media mix, manga, transmédialité, anime, Dragon Ball, fiction, Japon, jeux vidéo

\section{AUTEUR}

\section{BOUNTHAVY SUVILAY}

Université Paul-Valéry Montpellier 3, RIRRA21 et Université Paris Nanterre, CSLF-PHisTeM 